\title{
Miroir, mon beau miroir
}

\author{
Mirror, mirror, on the wall
}

Deux articles publiés dans ce numéro ${ }^{[1,2]}$ abordent la question de l'auto-évaluation en formation initiale des professionnels de la santé. Ces contributions illustrent en partie certaines évolutions récentes dans la manière de concevoir cette problématique et de l'aborder dans l'apprentissage en santé.

L'autorégulation des professions médicales est associée à un contrat social, au regard duquel les professions s'engagent notamment à assurer que leurs membres poursuivent leur apprentissage pour maintenir leurs compétences à jour ${ }^{[3]}$. Cette autorégulation, initialement formulée au niveau de la profession, s'est largement traduite en exigence d'autorégulation des individus, supposés capables de déterminer leurs besoins d'apprentissage et de choisir des activités de formation continue visant à les satisfaire. De nombreuses études en sciences de la santé, mais également dans d'autres domaines, ont mis à mal cette notion, mettant en évidence la faible concordance entre les auto-évaluations des individus et les évaluations externes ${ }^{[4-6]}$. Les raisons de ce phénomène sont multiples, incluant notamment le fonctionnement de la mémoire dans la genèse de ce type de jugement global, les besoins de protection de l'estime de soi, ainsi qu'un biais cognitif associé, selon lequel la majorité des individus se situent aux alentours du percentile 60-70 dans bon nombre de performances ${ }^{[4,7]}$.

Les recommandations actuelles découlant de ce constat mettent l'accent sur la nécessité d'une reconceptualisation de ce type d'auto-évaluation (self-assessment) comme une démarche de recueil délibérée de données pour s'évaluer (self-directed assessment seeking ${ }^{[7]}$. La réflexivité est une des formes que peut prendre cette démarche. Comme décrit dans l'article de Grevellec et Marchand ${ }^{[2]}$, tout particulièrement si elle est guidée par un enseignant ou un mentor, elle peut notamment permettre de rendre explicites les critères internes ou externes que les individus utilisent comme données pour s'évaluer. Ainsi, les réactions physiologiques telles que l'accélération du rythme cardiaque, peuvent suggérer une forte émotion, de l'anxiété par exemple, que les individus auront tendance à associer à un faible niveau de maîtrise de la tâche à effectuer ${ }^{[8]}$.

Cette démarche devrait inclure la recherche et l'analyse de données externes ${ }^{[7]}$. Celles-ci peuvent prendre des formes variées, comme les avis sollicités auprès des patients, de collègues ou de superviseurs $^{[9]}$. Il n'est toutefois pas évident de trouver des sources externes de rétro-action sur sa pratique. Si les patients peuvent être des juges pertinents pour des compétences relationnelles, ils ne peuvent se prononcer valablement sur le raisonnement clinique par exemple ; or il s'agit là d'une compétence-clé des professionnels de la santé. Les collègues, qui seraient plus à même d'émettre un jugement valide, ne sont que rarement sollicités. L'évaluation multi-sources ou à 360 degrés est une manière de formaliser ce processus $^{[10]}$. L'analyse des lettres de collègues consultés au sujet de patients en est une autre ${ }^{[9,11]}$.

Les praticiens peuvent pro-activement utiliser des évaluations standardisées telles que des tests de connaissance, proposés notamment par les associations professionnelles. Bien que limité aux compétences cognitives, ce type d'évaluation est une ressource intéressante. Des exemples de formation continue fondée sur des évaluations répétées voient d'ailleurs le jour ${ }^{[12]}$. Les technologies de l'information et les patients virtuels sont un autre exemple de 
ressources qui pourraient se développerencore davantage à l'avenir ${ }^{[13]}$.

En plus des difficultés liées à l'obtention de rétro-action (feedback), des études récentes mettent en évidence le fossé séparant la réception du feedback et son utilisation ${ }^{[14]}$. Les individus commencent en effet par comparer le feedback externe à leurs propres perceptions. L'incongruence entre les deux peut générer des émotions négatives, qui risquent de mener à des stratégies défensives visant à protéger l'estime de soi, telles que celle visant à discréditer la source du feedback externe, ce qui permet de la rejeter. La réflexivité permettrait à terme une assimilation du feedback et serait améliorée une fois encore par un guidage externe ${ }^{[14]}$.

Si l'auto-évaluation a été initialement étudiée dans le cadre de jugements globaux dans une perspective de choix d'activités d'apprentissage, elle se déploie également sous une forme plus fine dans la pratique autorégulée, processus appelé par Eva et Regehr self-monitoring ${ }^{[15]}$. En pratique professionnelle, cette forme d'auto-évaluation concerne par exemple la décision de vérifier la dose d'un médicament. Moulton et al. ont décrit les processus en jeu en salle d'opération lorsque le chirurgien reconnaît faire face à une difficulté et met en œuvre des stratégies pour la surmonter, notamment en modifiant le contexte (arrête de parler, demande d'éteindre la musique) pour augmenter sa concentration et lui permettre de poursuivre de manière délibérée plutôt qu'automatique ${ }^{[16]}$.

En formation initiale, cette forme d'auto-évaluation se manifeste dans l'évaluation de la certitude à une question d'examen ou d'une connaissance évoquée en séance d'apprentissage par problèmes, comme dans l'étude de Collard et al. ${ }^{[1]}$. Il semblerait que sous cette forme, l'auto-évaluation soit entachée de moins d'erreurs ${ }^{[17]}$. L'équipe d'Eva a par exemple montré que les étudiants pouvaient identifier des questions pour lesquelles ils manquaient de connaissances, mettant plus de temps pour y répondre ou différant leur réponse de manière appropriée ${ }^{[17,18]}$. Il n'empêche que cette forme d'auto-évaluation n'est pas parfaite, que les individus, particulièrement les moins performants, ont un certain nombre d'idées fausses (misconceptions) dont ils sont néanmoins certains $^{[18,19]}$.

En formation spécialisée ou continue, cette forme d'auto-évaluation entre en jeu par exemple lorsque le résident demande de l'aide à son superviseur. Là encore, il est important de mettre en garde les superviseurs à l'égard des imperfections de l'autoévaluation, même sous sa forme plus spécifique. $\mathrm{Si}$ des erreurs d'appréciation peuvent être à l'œuvre, entre auto-évaluation et action (par exemple appel à l'aide), il y a là encore un fossé, influencé notamment par des facteurs contextuels générant la crainte de paraître incompétent ou de déranger, y compris chez les praticiens ${ }^{[20,21]}$.

Ces réflexions amènent à concevoir l'auto-évaluation comme un élément parmi de multiples autres de l'apprentissage et de la pratique autorégulés ${ }^{[22,23]}$. Sans viser l'exhaustivité, nous en citerons quelquesuns pour illustrer la complexité de l'autorégulation.

L'intention d'apprendre et la fixation de buts d'apprentissage dépendent de l'autoévaluation, mais également d'autres facteurs, comme par exemple l'analyse, tant cognitive qu'affective, de la tâche. En formation initiale par exemple, les étudiants se fondent sur de multiples sources d'informations pour évaluer les tâches requises à la préparation de leurs examens ${ }^{[24]}$. Parmi d'autres facteurs déterminants, citons les buts de compétence (valorisant la démonstration aux autres de sa compétence et concevant donc l'erreur comme un échec, ou à l'inverse, valorisant l'apprentissage et concevant donc l'erreur comme source d'apprentissage).

L'engagement dans la tâche d'apprentissage fait appel à des stratégies cognitives (telles que la répétition ou l'élaboration de schémas ou de tableaux) et métacognitives (telles que l'auto-questionnement après avoir étudié un paragraphe pour vérifier l'acquisition de connaissances), qui toutes deux dépendent des savoirs des étudiants (portant sur le contenu de l'apprentissage mais également sur les stratégies d'apprentissage). Leur choix de stratégies cognitives et métacognitives dépendra aussi de leurs croyances épistémiques ${ }^{[25,26]}$. Il s'agit de croyances concernant le savoir. Les apprenants convaincus que le savoir en question est fixe et justifié par l'autorité, 
se contenteront par exemple de consulter une seule source considérée comme valide et chercheront à la mémoriser, tandis que ceux qui pensent que le savoir est issu d'une pondération de données issues de recherches dont les critères de validité doivent être évalués s'engageront dans un processus de confrontation et d'évaluation de multiples données. Enfin, la phase d'apprentissage requiert un contrôle de l'attention et de l'effort. Ainsi les étudiants peuvent modifier le contexte d'étude pour favoriser leur concentration, renforcer leurs représentations positives de la valeur de la tâche, réguler leurs éventuelles émotions négatives.

Enfin, l'évaluation de l'apprentissage comprend une nouvelle auto-évaluation, influencée par les émotions et par les phénomènes d'attribution, pour ne citer qu'eux.

Les modèles actuels mettent en évidence la complexité et le caractère dynamique des conduites autorégulées, mettant en œuvre des processus cognitifs, affectifs, volitionnels (c'est-à-dire relatifs à la manifestation de la volonté) et métacognitifs, tantôt conscients, tantôt inconscients ${ }^{[22,23]}$. Ceux-ci sont dépendants de facteurs individuels, mais également de facteurs contextuels et culturels. Les dispositifs pédagogiques mettant l'accent sur l'apprentissage de l'autorégulation ne peuvent donc plus se limiter à encourager et encadrer la pratique de l'auto-évaluation. Au vu de la complexité du phénomène et des moyens à disposition dans des institutions de formation de masse, le défi est certainement de taille.

$$
\begin{array}{r}
\text { Valérie DORY } \\
\text { Rédactrice associée } \\
\text { Mailto : valerie.dory@ mcgill.ca }
\end{array}
$$

\section{Références}

1. Collard A, Melot F, Bourguignon J-P. Intérêt d'un exercice centré sur la capacité d'auto-validation des connaissances au cours d'un dispositif d'apprentissage par problèmes. Pédagogie Médicale 2014;15: 73-89.

2. Grevellec C, Marchand C. Étude des autoévaluations des étudiants en soins infirmiers au décours d'expériences cliniques. Pédagogie Médicale 2014; 15:99-111.

3. Cruess SR, Johnston S, Cruess RL. "Profession": A Working Definition for Medical Educators. Teach Learn Med 2004;16:74-6.

4. Kruger J, Dunning D. Unskilled and unaware of it: how difficulties in recognizing one's own incompetence lead to inflated self-assessments. J Pers Soc Psychol 1999;77:1121-34.

5. Gordon MJ. A review of the validity and accuracy of self-assessments in health professions training. Acad Med 1991;66:762-9.

6. Davis DA, Mazmanian PE, Fordis M, et al. Accuracy of physician self-assessment compared with observed measures of competence: a systematic review. JAMA 2006;296:1094-102.

7. Eva KW, Regehr G. "I'll never play professional football" and other fallacies of self-assessment. J Contin Educ Health Prof 2008;28:14-9.

8. Bandura A. Self-Efficacy: The Exercise of Control. New York: Freeman, 1997.

9. Lockyer J, Armson H, Chesluk B et al. Feedback data sources that inform physician self-assessment. Med Teach 2011;33(2):e113-e20.

10. Lockyer J. Multisource feedback in the assessment of physician competencies. J Contin Educ Health Prof 2003;23:4-12.

11. Hoftvedt BO, Mjell J. Referrals: Peer review as continuing medical education. Teach Learn Med 1993;5:234-7.

12. Hornos EH, Pleguezuelos EM, Brailovsky CA, et al. The practicum script concordance test: an online continuing professional development format to foster reflection on clinical practice. J Contin Educ Health Prof 2013;33:59-66.

13. Cook DA, Erwin PJ, Triola MM. Computerized virtual patients in health professions education: a systematic review and meta-analysis. Acad Med 2010;85:1589-602.

14. Sargeant J, Mann K, Van der Vleuten C, Metsemakers J. Reflection: a link between receiving and using assessment feedback. Adv Health Sci Educ Theory Pract 2009;14:399-410.

15. Eva KW, Regehr G. Self-assessment in the health professions: a reformulation and research agenda. Acad Med 2005;80(10 Suppl):S46-S54.

16. Moulton CA, Regehr G, Mylopoulos M, MacRae HM. Slowing down when you should: a new model 
of expert judgment. Acad Med 2007;82(10 Suppl):S109-S16.

17. Eva KW, Regehr G. Knowing when to look it up: a new conception of self-assessment ability. Acad Med 2007;82(10 Suppl):S81-S4.

18. McConnell MM, Regehr G, Wood TJ, Eva KW. Selfmonitoring and its relationship to medical knowledge. Adv Health Sci Educ Theory Pract 2012;17(3):311-23

19. Dory V, Degryse J, Roex A, Vanpee D. Usable knowledge, hazardous ignorance - beyond the percentage correct score. Med Teach 2010;32:375-80.

20. Kennedy TJT, Regehr G, Baker GR, Lingard LA. "It's a cultural expectation..." The pressure on medical trainees to work independently in clinical practice. Med Educ 2009;43:645-53.

21. Leung A, Luu S, Regehr G, et al. "First, do no harm": balancing competing priorities in surgical practice. Acad Med 2012;87:1368-74.
22. Brydges R, Butler D. A reflective analysis of medical education research on self-regulation in learning and practice. Med Educ 2012;46:71-9.

23. Cosnefroy L. L'Apprentissage autorégulé: entre cognition et motivation. Bressoux P, editor. Grenoble: Presses universitaires de Grenoble, 2011.

24. Cilliers F, Schuwirth L, Herman N, Adendorff H, Van der Vleuten C A model of the pre-assessment learning effects of summative assessment in medical education. Adv Health Sci Educ Theory Pract 2012; 17:39-53.

25. Roex A, Degryse J. Introducing the concept of epistemological beliefs into medical education: the hot-air-balloon metaphor. Acad Med 2007;82: 616-20.

26. Knight LV, Mattick K. "When I first came here, I thought medicine was black and white": Making sense of medical students' ways of knowing. Soc Sci Med 2006;63:1084-96. 